\title{
On (Non) Applicability of a Mode-Truncation of a Damped Traveling String
}

\author{
RAJAB ALI MALOOKANI*, SAJAD HUSSAIN SANDILO*, AND ABDUL HANAN SHEIKH*
}

RECEIVED ON 06.04.2018 ACCEPTED ON 25.05.2018

\begin{abstract}
This study investigates a linear homogeneous initial-boundary value problem for a traveling string under linear viscous damping. The string is assumed to be traveling with constant speed, while it is fixed at both ends. Physically, this problem represents the vertical (lateral) vibrations of damped axially moving materials. The axial belt speed is taken to be positive, constant and small in comparison with a wave speed, and the damping is also considered relatively small. A two timescale perturbation method together with the characteristic coordinate's method will be employed to establish the approximateanalytic solutions. The damped amplitude-response of the system will be computed under specific initial conditions. The obtained results are compared with the finite difference numerical technique for justification. It turned out that the introduced damping has a significant effect on the amplitude-response. Additionally, it is proven that the mode-truncation is applicable for the damped axially traveling string system on a timescale of order $\varepsilon^{-1}$.
\end{abstract}

Key Words: Traveling String, Viscous Damping, Two Timescales, Truncation.

\section{INTRODUCTION}

$\mathrm{M}$ oving structures have vast applications in various engineering disciplines such as civil, mechanical and aerospace engineering. These systems represent many engineering devices, for instances; conveyor belts, elevator cables, chair lifts, pipes transporting liquids and gases, power transformation lines, and serpentine belts are few of them.Axially moving systems are mainly divided into two categories, that is, traveling string and EulerBernoulli beam. Axial speed and/or tension may excite the transversal vibrations of axially moving systems.
Vibrations (mainly lateral) in such devices have limited their applications. Over the last six decades, there has been vast research on the lateral vibrations of axially traveling strings or beams. Miranker [1] was the first who developed the mathematical equations of motion for laterals oscillations of axially accelerating string. For further studies on vibrations of string and beams, the reader is referred the papers [2-16]. To suppress the vibrations and noise in structures and machines, damping of string material is widely taken into consideration [17]. Gaiko [8] recently developed the

Authors E-Mail (rajab_ali31@yahoo.com, s.h.sandilo@quest.edu.pk, ah.sheikh@quest.edu.pk)

* Department of Mathematics \& Statistics, Quaid-e-Awam University of Engineering, Science \& Technology, Nawabshah, Pakistan.

This is an open access article published by Mehran University Research Journal of Engineering and Technology, Jamshoro under the CC by 4.0 International License. 
equations of motion for viscous damping of accelerating string with the help of extended Hamilton principle. Various analytical and numerical techniques were employed to investigate elastic and viscoelastic strings or beams under different operating conditions. Sandilo et. al. [10] examined the viscous damping via two timescales perturbation method. Gaiko and van Horssen [5] used the method of Laplace transform and two timescale perturbation method to investigate the transversal vibrations of traveling string with the boundary damping. Fung et. al. [11] computed the response of a moving viscoelastic string by means of Galerkin discretization and finite difference numerical technique. Gahyesh [18] examined the steady state response of viscoelastic string through Routh-Hurwitz criterion. Shahruz [19] analyzed the stability of a nonlinear axially moving string with Kelvin-Voigt damping through Lyapunov function. Chen, et. al. [20] examined the effects of viscous damping at one end of the boundary under the effect of various axial string velocities in axially moving string via finite element method. Zhang and $\mathrm{Zu}$ [21] examined the free transverse vibrations in viscoelastic axially moving string via method of multiple scales. Maitlo et. al. [14] investigated the damped axially moving system via perturbation method together with the Fourier-series expansion method. However, only few modes were taken in [13] to examine the behavior of the amplitude-response of the damped traveling string system.

In continuation of [14], this study will compute the all mode-responses without any truncation via the combination of the method of characteristic coordinates and two timescales perturbation method. In addition, the (non)applicability of mode-truncation for damped traveling string systems will also be discussed. The results obtained through the proposed technique will also be validated via finite difference numerical technique.

\section{EQUATIONS OF MOTION}

Consider a uniform axially moving string of mass density $\rho$, initial tension $\mathrm{T}_{0}$, the viscous damping parameter $\beta$ and uniform axial transport speed $\mathrm{V}$ that travels between a pair of pulleys separated by a distance $\mathrm{L}$ and is assumed to be fixed at both ends, i.e. at $\mathrm{x}=0$ and $\mathrm{x}=\mathrm{L}$ as shown in Fig. 1. The model is restricted under the following assumptions: (i) only the vertical displacement $u(x, t)$ is considered, where $x$ spatial coordinate along the string, and $t$ is the time. (ii) The parameters $\rho, \mathrm{V}, \mathrm{T}_{0}$ and $\beta$ are all taken as positive constants. The gravity and other external forces are not taken into consideration. Based on the above mentioned assumptions, the governing equations describing the vertical (transversal) oscillations of the traveling string with linear viscous damping are derived by using the Hamilton principle $[8,13]$ and are given by:

$\rho\left(u_{t t}+2 V u_{x t}+V^{2} u_{x x}\right)-T_{0} u_{x x}+\beta\left(u_{t}+V u_{x}\right)=0 \quad 0<x<L, t \geq 0$

with the boundary conditions:

$\mathrm{u}(0, \mathrm{t})=\mathrm{u}(\mathrm{L}, \mathrm{t})=0, \mathrm{t} \geq 0$

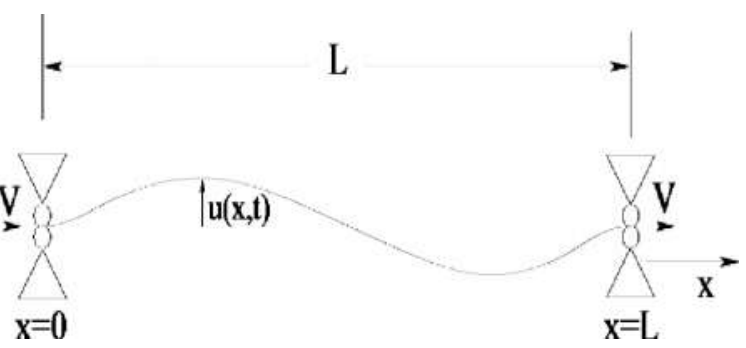

FIG. 1. SCHEMATIC MODEL OF A TRAVELING STRING

Mehran University Research Journal of Engineering \& Technology, Volume 38, No. 2, April, 2019 [p-ISSN: 0254-7821, e-ISSN: 2413-7219] 
and initial conditions:

$\mathrm{u}(\mathrm{x}, 0)=\mathrm{u}_{0}(\mathrm{x}), \mathrm{u}_{\mathrm{t}}(\mathrm{x}, 0)=\mathrm{u}_{1}(\mathrm{x}), \quad 0<\mathrm{x}<\mathrm{L}$

where $\mathrm{u}_{0}$ is the initial displacement and $\mathrm{u}_{1}$ is the initial speed of the system.

To put the Equations (1-3) in a dimensionless form, the following non-dimensional quantities are taken into consideration:

$\widetilde{\mathrm{x}}=\frac{\mathrm{x}}{\mathrm{L}}, \widetilde{\mathrm{u}}=\frac{\mathrm{u}}{\mathrm{L}}, \widetilde{\mathrm{t}}=\frac{\mathrm{tc}}{\mathrm{L}}, \widetilde{\mathrm{V}}=\frac{\mathrm{L}}{\mathrm{c}}, \widetilde{\beta}=\frac{\beta \mathrm{L}}{\rho \mathrm{c}}, \widetilde{\mathrm{u}}_{0}=\frac{\mathrm{u}_{0}}{\mathrm{~L}}, \widetilde{\mathrm{u}}_{1}=\frac{\mathrm{u}_{1}}{\mathrm{c}}$

where $c=\sqrt{\frac{T_{0}}{\rho}}$ is a wave speed.

Thus, the Equations (1-3) in dimensionless form becomes,

$\mathrm{u}_{\mathrm{tt}}+2 \mathrm{Vu}_{\mathrm{xt}}+\left(\mathrm{V}^{2}-1\right) \mathrm{u}_{\mathrm{xx}}+\beta\left(\mathrm{u}_{\mathrm{t}}-\mathrm{Vu}_{\mathrm{x}}\right)=0 \quad 0<\mathrm{x}<1, \mathrm{t} \geq 0$

with boundary conditions

$\mathrm{u}(0, \mathrm{t})=\mathrm{u}(1, \mathrm{t})=0, \quad \mathrm{t} \geq 0$

with initial conditions

$u(x, 0)=u_{0}(x), u_{t}(x, 0)=u_{1}(x), \quad 0 \leq x \leq 1$

The hats describing the non-dimensional quantities are neglected in Equations (5-7), and from now on. In the subsequent sections, the Equations (5-7) will be solved through the combination of two timescales perturbation method and the method of characteristics.

\section{CONSTRUCTION OF FORMAL APPROXIMATIONS}

In this section, the formal asymptotic approximations for the homogeneous linear initial-boundary value problem Equations (5-7) will be developed via the combination of the method of two timescales perturbation and the method of characteristic coordinates. For further details of this technique, the reader is referred to [2,6-7,22-23]. The low axial belt speed Vof the string is considered in comparison to wave speed cand $0(\varepsilon)$, that is, $\mathrm{V}=0(\varepsilon)$, where $\varepsilon(0<\varepsilon<<$ 1 ) is a book keeping parameter. It is also presumed that $\beta \mathrm{L}$ is small compared to $\rho c$ and $0(\varepsilon)$, i.e. $\beta=0(\varepsilon)$. Based on these assumptions, Equations (5-7) can be written as follows:

$u_{t t}-u_{x x}=-\varepsilon\left(2 v_{x t}+\beta u_{t}\right)-\varepsilon^{2}\left(V^{2} u_{x x}+\beta V u_{x}\right) \quad 0<x<1, t \geq 0$

where the boundary and initial conditions are given as in Equations (6-7). In order to investigate the initial boundary value problem (8), a two timescales perturbation technique will be employed. However, we encounter several computational complexities whenever we employ a Fourier expansion method of the unperturbed solution as were noticed in [13]; the equations of motion (8) is alternatively investigated by means of the characteristic variables $\sigma=\mathrm{x}-\mathrm{t}$ and $\xi=\mathrm{x}+\mathrm{t}$. In this method, we will replace the initial-boundary value problem (8) by an initial-value problem. This replacement requires the extension of the dependent variable $u$ and its derivatives and the initial values in $\mathrm{x}$ to two-periodic odd functions. Since the terms $u_{x t}$ and $u_{x}$ in Equation (8) are not odd; in order to make them odd, we multiply these terms with the Fourier-sine series:

$H(x)=\sum_{n=1}^{\infty} \frac{4}{(2 n-1) \pi} \sin ((2 n-1) \pi x)$

With the multiplication of $\mathrm{H}(\mathrm{x})$ in Equation (8), the equation becomes

$\mathrm{u}_{\mathrm{tt}}-\mathrm{u}_{\mathrm{xx}}=-\varepsilon\left(2 \mathrm{Vu}_{\mathrm{xt}} \mathrm{H}(\mathrm{x})=\beta \mathrm{u}_{\mathrm{t}}\right)-\varepsilon^{2}\left(\mathrm{~V}^{2} \mathrm{u}_{\mathrm{xx}}+\beta \mathrm{Vu}_{\mathrm{x}} \mathrm{H}(\mathrm{x})\right), 0<\mathrm{x}<1, \mathrm{t} \geq 0$ (10) 
By applying the perturbation method to Equation (10), the solution is assumed in the form

$\mathrm{u}(\mathrm{x}, \mathrm{t})=\mathrm{v}(\sigma, \xi, \tau)$

where $\sigma=\mathrm{x}-\mathrm{t}, \xi=\mathrm{x}+\mathrm{t}$, and $\tau=\varepsilon \mathrm{t}$.

The introduction of $\sigma, \xi$ and $\tau$ leads to the following transformation:

$\mathrm{u}_{\mathrm{t}}=-\mathrm{v}_{\sigma}+\mathrm{v}_{\xi}+\varepsilon \mathrm{v}_{\tau}, \mathrm{u}_{\mathrm{tt}}=\mathrm{v}_{\sigma \sigma}+\mathrm{v}_{\xi \xi}-2 \mathrm{v}_{\sigma \xi}+2 \varepsilon\left(\mathrm{v}_{\xi \tau}-\mathrm{v}_{\sigma \tau}\right)$

$\mathrm{u}_{\mathrm{x}}=\mathrm{v}_{\sigma}+\mathrm{v}_{\xi}, \mathrm{u}_{\mathrm{xx}}=\mathrm{v}_{\sigma \sigma}+\mathrm{v}_{\xi \xi}+2 \mathrm{v}_{\sigma \xi}, \mathrm{u}_{\mathrm{x} \tau}=-\mathrm{v}_{\sigma \sigma}+\mathrm{v}_{\xi \xi}+\varepsilon\left(\mathrm{v}_{\sigma \tau}+\mathrm{v}_{\xi \tau}\right)$

Plugging Equations (11-12) into Equation (10), we obtain:

$-4 \mathrm{v}_{\sigma \xi}=\varepsilon\left[2 \mathrm{v}_{\sigma \tau}-2 \mathrm{v}_{\xi \tau}+2 \mathrm{VH}\left(\frac{\xi+\sigma}{2}\right)\left(\mathrm{v}_{\sigma \sigma}-\mathrm{v}_{\xi \xi}\right)+\beta\left(\mathrm{v}_{\sigma}-\mathrm{v}_{\xi}\right)\right]+\mathrm{O}\left(\varepsilon^{2}\right)$

Further, we assume that the function $\mathrm{v}(\sigma, \xi, \tau)$ can be expanded as:

$\mathrm{v}(\sigma, \xi, \tau)=\mathrm{v}_{0}(\sigma, \xi, \tau)+\varepsilon \mathrm{v}_{1}(\sigma, \xi, \tau)+\mathrm{O}\left(\varepsilon^{2}\right)$

Plugging Equation (14) into Equation (13), and comparing the terms of order $\varepsilon^{0}$ and $\varepsilon^{1}$, we obtain the $O(1)$ and $O(\varepsilon)$ problem below:

$\mathrm{O}(1): \begin{cases}-4 \mathrm{v}_{0 \sigma \xi}=0 & -\infty<\sigma<\xi<\infty, \tau>0 \\ \mathrm{v}_{0}(\sigma, \sigma, 0)=\mathrm{u}_{0}(\sigma) & -\infty<\sigma=\xi<\infty, \tau=0 \\ -\mathrm{v}_{0 \sigma}(\sigma, \sigma, 0)+\mathrm{v}_{0 \xi}(\sigma, \sigma, 0)=\mathrm{u}_{1}(\sigma) & -\infty<\sigma=\xi<\infty, \tau=0\end{cases}$

and

$\mathrm{O}(\varepsilon):\left\{\begin{array}{lc}-4 \mathrm{v}_{1 \sigma \xi \xi}=2 \mathrm{v}_{0 \sigma \tau}-2 \mathrm{v}_{0 \xi \tau}+2 \mathrm{VH}\left(\frac{\xi+\sigma}{2}\right)\left(\mathrm{v}_{0 \sigma \sigma}-\mathrm{v}_{0 \xi \xi}\right)+\beta\left(\mathrm{v}_{0 \sigma}-\mathrm{v}_{0 \xi}\right) & -\infty<\sigma<\xi<\infty, \tau>0 \\ \mathrm{v}_{1}(\sigma, \sigma, 0)=0 & -\infty<\sigma=\xi<\infty, \tau=0 \\ -\mathrm{v}_{1 \sigma}(\sigma, \sigma, 0)+\mathrm{v}_{1 \xi}(\sigma, \sigma, 0)=\mathrm{v}_{0 \tau}(\sigma, \sigma, 0) & -\infty<\sigma=\xi<\infty, \tau=0\end{array}\right.$

It should be noticed that the term $\varepsilon \mathrm{v}_{1}$ in Equation (14) is assumed to be small in comparison to the term $v_{o}$ and that the expansion is uniform in the sense that the ratio $\varepsilon \mathrm{v}_{1} / \mathrm{v}_{\mathrm{o}}$ is $\mathrm{O}(1)$ for all t. For the solution Equation (14) to be valid for times as large as $\mathrm{O}\left(\varepsilon^{-1}\right)$; the terms in $\mathrm{v}_{1}$ which, grow linearly with respect to $t$ must be eliminated.The general solution of $\mathrm{O}(1)$-problem is given by:

$\mathrm{v}_{0}(\sigma, \xi, \tau)=\mathrm{f}_{0}(\sigma, \tau)+\mathrm{g}_{0}(\xi, \tau)$

where $\tau$ appears only parametrically to this order of approximation, and the functions $\mathrm{f}_{0}$ and $\mathrm{g}_{0}$ satisfy the initial conditions:

$\mathrm{f}_{0}(\sigma, 0)+\mathrm{g}_{0}(\sigma, 0)=\mathrm{u}_{0}(\sigma)$ and $-\mathrm{f}_{0}^{\prime}(\sigma, 0)+\mathrm{g}_{0}^{\prime}(\sigma, 0)=\mathrm{u}_{1}(\sigma)$

In addition to this, the two-periodic odd extension of the dependent variable $\mathrm{u}$ imply that the functions $\mathrm{f}_{0}$ and $\mathrm{g}_{0}$ also have to satisfy $\mathrm{g}_{0}(\sigma, \tau)=-\mathrm{f}_{0}(\sigma, \tau)$ and $\mathrm{f}_{0}(\sigma, \tau)=\mathrm{f}_{0}(\sigma+2$, $\tau),-\infty<\sigma<\infty$, and $\tau \geq 0$. The unknown behavior of $f_{0}$ and $g_{0}$ with respect to $\tau$ will be used so that $\mathrm{v}_{1}$ does not involve secular terms. From Equation (16), $v_{1 \sigma}$ and $v_{1 \xi}$ can easily be obtained by integration, respectively, with respect to

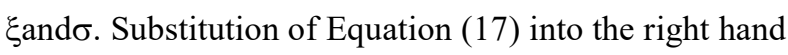
side of Equation (16) gives:

$-4 \mathrm{v}_{1 \sigma \xi}=2 \mathrm{f}_{0 \sigma \tau}-2 \mathrm{~g}_{0 \xi \tau}+2 \mathrm{VH}\left(\frac{\xi+\sigma}{2}\right)\left(\mathrm{f}_{0 \sigma \sigma}-\mathrm{g}_{0 \xi \xi}\right)+\beta\left(\mathrm{f}_{0 \sigma}-\mathrm{g}_{0 \xi}\right)$

Integration of Equation (18) with respect to $\xi$ yields:

$$
\begin{aligned}
& 4 \mathrm{v}_{1 \sigma}(\sigma, \xi, \tau)=4 \mathrm{v}_{1 \sigma}(\sigma, \sigma, \tau)-(\xi-\sigma)\left(2 \mathrm{f}_{0 \sigma \tau}+\beta \mathrm{f}_{0 \sigma}\right) \\
& -\int_{\sigma}^{\xi}\left[\left(2 \mathrm{~g}_{0 \xi \tau}+\beta \mathrm{g}_{0 \xi}\right)-2 \mathrm{VH}\left(\frac{\xi+\sigma}{2}\right)\left(\mathrm{f}_{0 \sigma \sigma}-\mathrm{g}_{0 \xi \xi}\right)\right] \mathrm{d} \xi+\mathrm{F}(\sigma, \tau)
\end{aligned}
$$

where the function $\mathrm{F}(\sigma, \tau)$ is a constant of integration and to be determined by the condition on $\mathrm{v}_{1}$. In Equation (19), it can be observed that the term $\xi-\sigma=2 \mathrm{t}$ is of order $\varepsilon^{-1}$. So, $\mathrm{v}_{1 \sigma}$ will be of $\mathrm{O}\left(\varepsilon^{-1}\right)$ unless the terms linear in $\varepsilon-\sigma$ (that is, of order $\varepsilon^{-1}$ are eliminated. It turns out that both $\mathrm{v}_{1 \sigma}$ and $v_{1 \xi}$ is of $\mathrm{O}(1)$ on a timescale of $\mathrm{O}\left(\varepsilon^{-1}\right)$ if the functions $\mathrm{f}_{0}$ and $\mathrm{g}_{0}$ satisfy the following conditions: 
$2 \mathrm{f}_{0 \sigma \tau}+\beta \mathrm{f}_{0 \sigma}=0$

and

$-2 \mathrm{~g}_{0 \xi \tau}-\beta \mathrm{g}_{0 \xi}=0$

The Equation (20) and Equation (21) are equivalent. The solution of Equation (20) subject to the initial conditions:

$\mathrm{f}_{0 \sigma}(\sigma, 0)=\frac{1}{2}\left(\mathrm{u}_{0}^{\prime}(\sigma)-\mathrm{u}_{1}(\sigma)\right)$

is

$\mathrm{f}_{0 \sigma}(\sigma, \tau)=\frac{1}{2}\left[\mathrm{u}_{0}^{\prime}(\sigma)-\mathrm{u}_{1}(\sigma)\right] \exp \left(\frac{-\beta \tau}{2}\right)$

The integration of Equation (23) with respect to $\sigma$ yields

$\mathrm{f}_{0}(\sigma, \tau)=\frac{1}{2}\left[\mathrm{u}_{1}(\sigma)-\int_{0}^{\sigma} \mathrm{u}_{1}(\sigma) \mathrm{d} \sigma\right] \exp \left(\frac{-\beta \tau}{2}\right)$

\section{SPECIFIC INITIAL CONDITIONS}

$\mathrm{u}(\mathrm{x}, 0)=2 \sin (\pi \mathrm{x}), \mathrm{u}_{\mathrm{t}}(\mathrm{x}, 0)=0$

With above initial conditions, the Equation (23) becomes

$f_{0}(\sigma, \tau)=\sin (\pi \sigma) \exp \left(\frac{-\beta \tau}{2}\right)$

Since $\mathrm{g}_{0}(\sigma, \tau)=-\mathrm{f}_{0}(-\sigma, \tau)$, so the first order approximation is obtained as:

$\mathrm{v}_{0}(\sigma, \xi, \tau)=\mathrm{f}_{0}(\sigma, \tau)-\mathrm{f}_{0}(-\xi, \tau) \equiv[\sin (\pi \sigma)+\sin (\pi \xi)] \exp \left(\frac{-\beta \tau}{2}\right)+\mathrm{O}(\varepsilon)$

Thus, the close form solution of initial-boundary value problem Equations (1-3) valid on $\mathrm{t}=\mathrm{O}\left(\varepsilon^{-1}\right)$ is expressed as:

$\mathrm{u}(\mathrm{x}, \mathrm{t})=\mathrm{v}_{0}(\mathrm{x}, \mathrm{t}, \tau)+\mathrm{O}(\varepsilon)=[\sin (\pi(\mathrm{x}-\mathrm{t}))+\sin (\pi(\mathrm{x}+\mathrm{t}))] \exp \left(\frac{-\beta \tau}{2}\right)+\mathrm{O}(\varepsilon)$ $\mathrm{u}(\mathrm{x}, 0)=\sin (\pi \mathrm{x}), \mathrm{u}_{\mathrm{t}}(\mathrm{x}, 0)=0.5 \sin (\pi \mathrm{x})$

The first order approximations is:

$\mathrm{u}(\mathrm{x}, \mathrm{t})=\left[\left\{0.5 \sin (\pi \sigma)+\frac{0.25}{\pi} \cos (\pi \sigma)\right\}+\left\{0.5 \sin (\pi \xi)-\frac{0.25}{\pi} \cos (\pi \xi)\right\}\right] \exp \left(\frac{-\beta \tau}{2}\right)+O(\varepsilon)$

where $\sigma=\mathrm{xt}, \xi=\mathrm{x}+\mathrm{t}$

\section{RESULTS AND DISCUSSIONS}

The behavior of the approximate-analytic solution $\mathrm{v}_{\mathrm{o}}$ and the numerical solution $u$ of the Equations (1-3) in terms of amplitude-response under two different initial conditions are shown, in Figs. 2-3. In Fig. 2(a-b), the amplitude-response is computed with the harmonically varying initial displacement and zero initial velocity (that is, $\left.u(x, 0)=2 \sin (\pi x), u_{t}(x, 0)=0\right)$. While, Fig. 3(a-b) exhibits the amplitude-response of the system with the initial displacement: $\mathrm{u}_{0}(\mathrm{x})=\sin (\pi \mathrm{x})$ and the initial velocity: $u_{1}(x)=0.5 \sin (\pi x)$. It is shown in Figs. $2-3$ that the amplitude-response of the system is damped out so rapidly as the damping rate increases in the system. Furthermore, the reasonable agreement between the approximate-analytic and numerical solutions can be witnessed in Figs. 2-3.

\section{CONCLUSION}

In this study, we examined the transverse (lateral) vibrations of a damped traveling string. It is assumed that the string is traveling between a pair of pulleys under constant speed. The non-vibration boundary conditions are taken into consideration. The approximateanalytic solutions are constructed via the combination of two timescales perturbation method and the method of characteristic coordinates. The explicit formal approximations are computed in terms of the amplitude- 


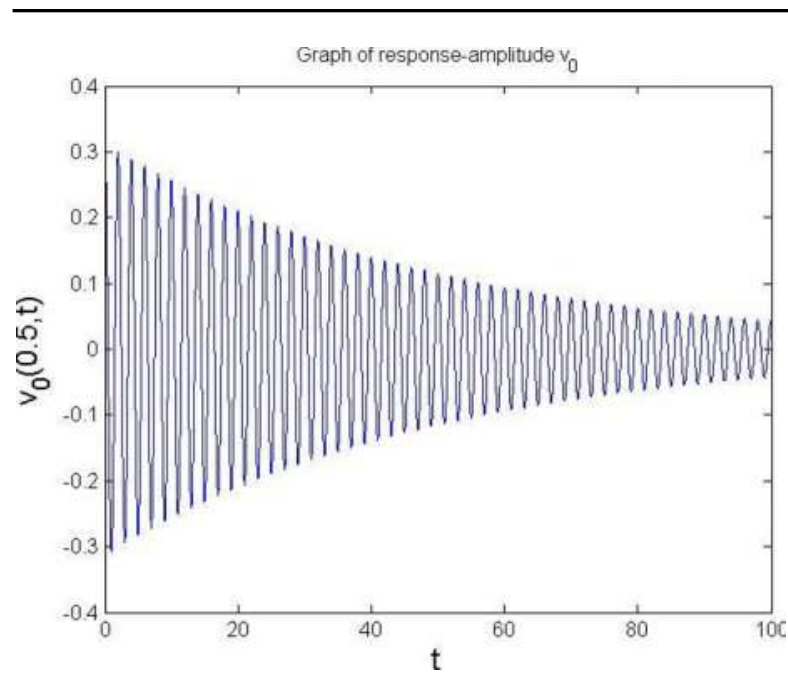

(a) $v_{0}$ (APPROXIMATE-ANALYTIC SOLUTION)

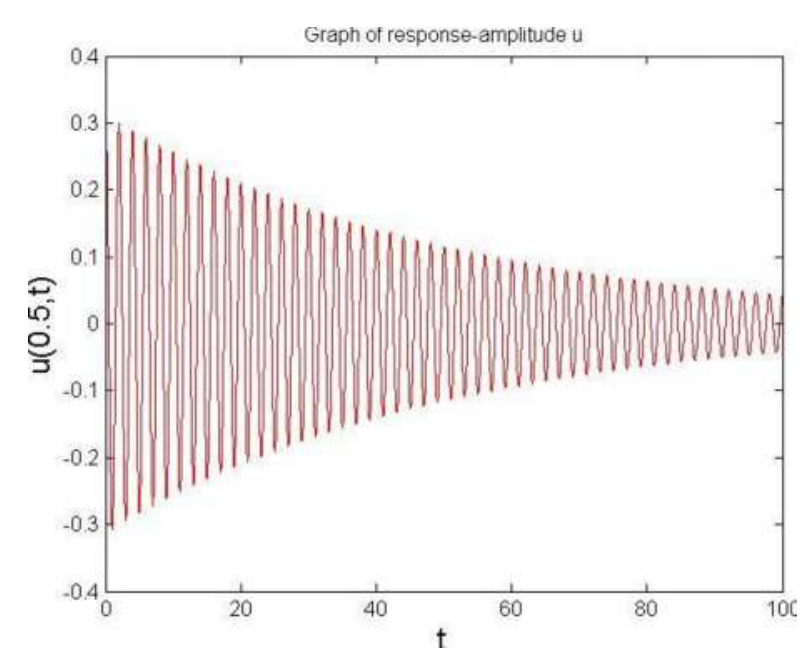

(b) $u$ (NUMERICAL SOLUTION) WITH $u_{0}(x)=2 \sin (\pi x)$ AND $u_{1}(x)=0$, for $\varepsilon=0.01, V=2, \beta=4$

FIG. 2. AMPLITUDE-RESPONSE

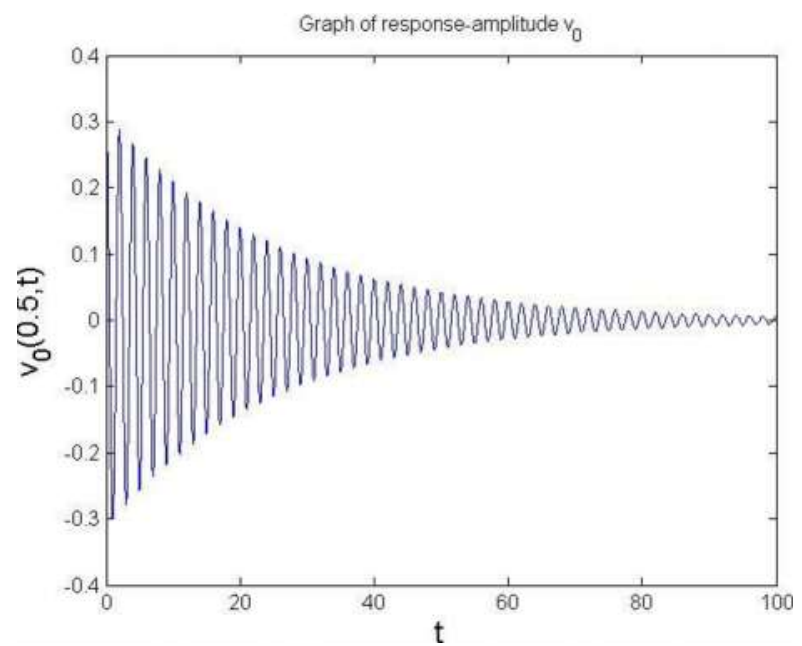

(a) $v_{0}$ (APPROXIMATE-ANALYTIC SOLUTION)

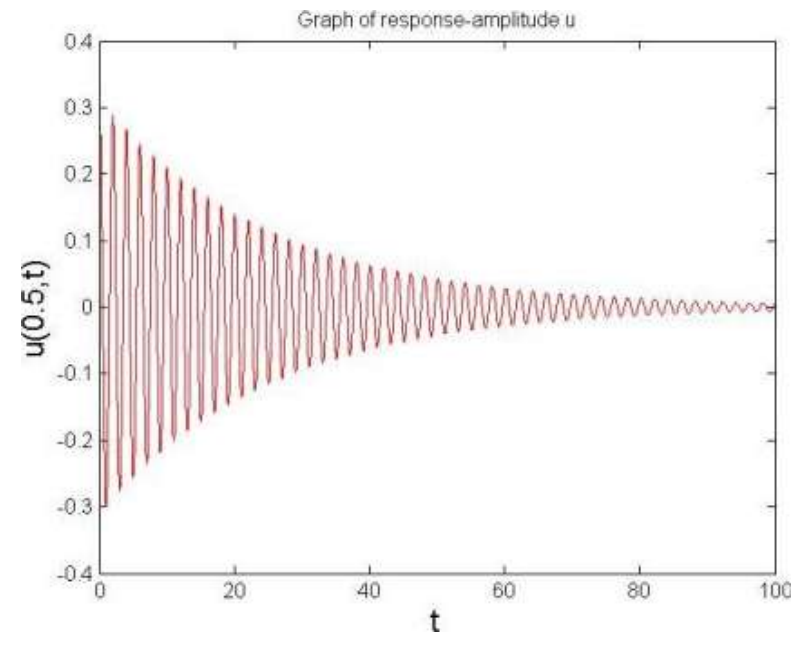

(b) $u$ (NUMERICAL SOLUTION) WITH $u_{0}(x)=\sin (\pi x)$ AND $u_{1}(x)=0.5 \sin (\pi x)$ for $\varepsilon=0.01, V=5, \beta=8$

FIG. 3. AMPLITUDE-RESPONSE

response subject to the two specific initial conditions. Under certain parameter values, it turned out that the amplitude response is damped out with the increasing values of damping parameter. Moreover, the amplituderesponse of individual modes in a traveling string was computedand shown as damped out. However, in this paper, the amplitude-response of the system in explicitform has been computed and shown to be damped out.
It is concluded that the mode-truncation is also possible for a damped traveling string.

\section{ACKNOWLEDGMENT}

The authors wish to express their gratitude towards the unknown reviewers for their valuable comments and suggestions, which indeed helped us to improve our manuscript. 


\section{REFERENCES}

[1] Miranker, W.L., "The Wave Equation in a Medium in Motion”, IBM Journal of Research and Development, Volume 4, No.1, pp. 36-42, 1960.

[2] Malookani, R.A., and van Horssen, W.T., "On Resonances and the Applicability of Galerkin'sTruncation Method for an Axially Moving String with Time-Varying Velocity", Journal of Sound and Vibration, Volume 344, pp. 1-17, 2015.

[3] Malookani, R.A., and van Horssen, W.T., "On the Asymptotic Approximation of the Solution of an Equation for a Non-Constant Axially Moving String”, Journal of Sound and Vibration, Volume 367, pp. 203-218, 2016.

[4] Gaiko, N.V., and van Horssen, W.T., "On the Transverse, Low Frequency Vibrations of a Traveling String with Boundary Damping", Journal of Vibration and Acoustics, Volume 137, No. 4, pp. 41004-41014, 2015.

Sandilo, S.H., and van Horssen, W.T., "On a Cascade of Auto-Resonances of an Elevator Cable System”, Nonlinear Dynamics, Volume 80, No. 3, pp. 1613-1630, 2015 .

[6] Chikwendu, C., and Kevorkian, J., "A Perturbation Method for Hyperolic Equations with Smal INonlinearities", SIAM Journal on Applied Mathematics, Volume 22, No. 2, pp. 235-258, 1972.

Darmawijoyo, van Horssen, W.T., and Celement, P.H.,"On a Rayleigh Wave Equation with Boundary Damping", Nonlinear Dynamics, Volume 33, No. 4, pp. 399-429, 2003.

[8] Gaiko, N.V., "Transverse Waves and Vibrations in Axially Moving Continua", Ph.D.Thesis, Delft University of Technology, 2017.

[9] Sandilo, S.H., Malookani, R.A., and Shaikh A.H., "On Oscillations of an Axially Translating Tensioned Beam under Viscous Damping", Science International, Volume28, No. 4, pp. 4123-4127, Lahore, Pakistan, 2016.
[10] Fung, R.F., Huang, J.S., and Chen, Y.C., "The Transient Amplitude of the Viscoelastic Traveling String: An Integral Constitutive Law", Journal of Sound and Vibration, Volume 201, No. 2, pp. 153-167, 1997.

[11] Mahalingam, S., "Transverse Vibrations of Power Transmission Chains”, British Journal of Applied Physics, Volume 8, No. 4, pp. 145-148, 1957.

[12] Sack, R.A., "Transverse Oscillations in Traveling Strings", British Journal of Applied Physics, Volume 5, No. 6, pp. 224-226, 1954.

[13] Maitlo, A.A., Sandilo, S.H., Shaikh, A.H., Malookani, R.A., and Qureshi, S.,"On Aspects of Viscous Damping for an Axially Transporting String", Science International, Volume 28, No. 4, pp. 3721-3727, Lahore, Pakistan, 2016.

[14] Sandilo, S.H., and van Horssen, W.T., "On Boundary Damping for an Axially Moving Tensioned Beam", Journal of Vibration and Acoustics, Transactions of the ASME, Volume 134, No. 1, pp. 11005-11013, 2012.

[15] Suweken, G., and van Horssen, W.T., "On the Transversal Vibrations of a Conveyor Belt with a Low and Time-Varying Velocity Part-I: The String-Like Case", Journal of Sound and Vibration, Volume 264, No. 1, pp. 117-133, 2003.

[16] Ponomareva, S.V., and van Horssen, W.T.,"On the Transversal Vibrations of an Axially Moving String with a Time-Varying Velocity", Nonlinear Dynamics, Volume 50, No. 1-2, pp. 315-323, 2007

[17] Ghayesh, M.H., "Nonlinear Transversal Vibration and Stability of an Axially Moving Viscoelastic String Supported by a Partial Viscoelastic Guide", Journal of Sound and Vibration Volume 314, No. 3-5, pp. 757-774, 2008.

[18] Shahruz, S.M., "Stability of a Nonlinear Axially Moving String with the Kelvin-Voigt Damping", Journal of Vibration and Acoustics, Volume 131, No. 1, pp. 14501-14505, 2009. 
[19] Chen, W.E., and Ferguson, N.S., "Analysis of Energy Dissipation in an Elastic Moving String with a Viscous Damper at One End", Journal of Sound and Vibration, Volume 333, No. 9, pp. 2556-2570, 2014.

[20] Zhang, L., and Zu, J.W., "Nonlinear Vibrations Viscoelastic Moving Belts, Part-1: Free Vibration Analysis", Journal of Sound and Vibration, Volume 216, No. 1, pp. 75-91, 1998.
[21 Jones, D.I., "Handbook of Viscoelastic Vibration Damping", John Wiley and Sons, New York, 2001.

[22] Nayfeh, A.H., "Perturbation Methods", John Wiley and Sons, New York, 2000.

[23] Kevorkian, J., and Cole, J.D., "Multiple Scale and Singular Perturbation”, Springer, 1996. 